\title{
Wachsamkeit als oberstes Prinzip
}

\section{Die Diagnose gefäßbedingter chronischer Wunden ist nicht schwierig. Trotzdem bleiben viele Patienten so lange unbehandelt, bis eine Amputation unvermeidbar ist.}

Schmerz, übler Geruch und ein abschreckendes Erscheinungsbild - Ulcus cruris, eine chronische, nicht heilende Wunde am Bein tritt bei der älteren Bevölkerung immer häufiger auf. Experten schätzen, dass Zehntausende betroffen sind.

Das Leiden verläuft langsam und wird besonders von älteren Betroffenen unterschätzt, aus Scham verschwiegen oder einfach nicht bemerkt. Zu spät wird ein Spezialist aufgesucht, in schweren Fällen führt das zur Amputation. Vorsorgemaßnahmen bei Risikogruppen und die rechtzeitige Therapie könnten dies verhindern.

\section{Jährlich Tausende Amputationen}

Es gibt keine einheitliche Definition, ab wann eine Wunde als chronisch zu bezeichnen ist. Vier bis sechs Wochen werden genannt. Prof. Dr. Franz Trautinger, Kampagnensprecher „Chronische Wunde“, Gefäßforum Österreich (GFÖ), möchte lieber wie folgt definieren: „Jede Wunde, die nicht normal abheilt". Besonders betroffen sind Menschen ab 65, Raucher und vor allem Diabetiker.

Gefäßbedingte chronische Wunden werden schätzungsweise bei einem Prozent der Bevölkerung - das wären in Österreich rund 85.000 Personen - viel zu spät behandelt, was zu einer Zunahme der Amputationen von 2002 bis 2006 um knapp elf Prozent auf jährlich 2.562 Eingriffe führte. Laut Doz. Dr. Afshin Assadian, Wissenschaftlicher Sprecher des GFÖ, sollte man die Chance wahrnehmen, eine Vorsorgeuntersuchung für Gefäßerkrankungen bei sich und vor allem bei älteren Familienmitgliedern durchführen zu lassen. Die ärztliche Diagnose ist nämlich nicht schwierig.

\section{Diagnose}

„Das dauert fünf Minuten und man braucht dazu kein CT und kein MRT“, sagt Assadian. „Fünf Fragen zu den Risikofaktoren (Diabetes, Bluthochdruck, Rauchen, Hypercholesterinämie, Hyperlipidämie), ein Blick auf die Füße und ein Griff: Fühlt sich das Bein kalt an bzw. kälter als das andere?"
In den meisten Fällen ist eine venöse Durchblutungsstörung am Bein die Ursache. Die häufigste Lokalisation ist der Unterschenkel, bevorzugt der Innenknöchel. Arterielle Ulcera treten bei älteren Patienten mit klassischen Risikofaktoren für arterielle Verschlusskrankheiten auf, meist an Zehenendgliedern oder an Druckpunkten von Schuhen.

Der diabetische Fuß ist eine besonders gefährliche Form der chronischen Wunde. „Die Wahrscheinlichkeit für einen Diabetiker, im Laufe seines Lebens eine Fußwunde zu bekommen, liegt bei etwa 25 Prozent", sagt Assadian. „Diese Wunden sind gefährliche Erkrankungen, die sehr häufig in der Amputation von Zehen, dem Fuß, manchmal sogar dem ganzen Bein enden." Die Ursache ist hier meist eine Kombination von Durchblutungsstörung und Nervenschädigung. Diese Neuropathie ist auch der Grund, warum gerade diabetische Wunden oft sehr spät erkannt werden. Die genannten Formen von Ulcus cruris können auch als Mischformen auftreten und werden insbesondere dann gefährlich, wenn es zur Wundinfektion kommt.

Andere, eher seltenere Ursachen sind Krebserkrankungen und Infektionen durch Bakterien, Viren oder Pilze.

\section{Interdisziplinarität}

„Wir haben ein sehr gutes Gesundheitssystem. Was bei uns ein bisschen fehlt, ist die Zusammenarbeit", stellt Assadian fest. Die Kooperation von Hausärzten, Gefäßspezialisten, Internisten, Dermatologen und Orthopäden ist ihm ein Anliegen. Modelle der integrierten Versorgung, wie es sie in Deutschland gibt, wären wünschenswert.

„Die Gefäßmedizin hat enorme Fortschritte gemacht", sagt Trautinger. Gemeint sind nicht nur minimalinvasive Operationsmethoden, sondern auch neue Verbandsmaterialien, die die Wunde nicht austrocknen. In der Behandlung von venösen Beingeschwüren hat neben der Reinigung und Versorgung der Wunde die Kompressionsbehandlung weiterhin hohen Stellenwert, die laut Trautinger „viel zu wenig angewendet wird."

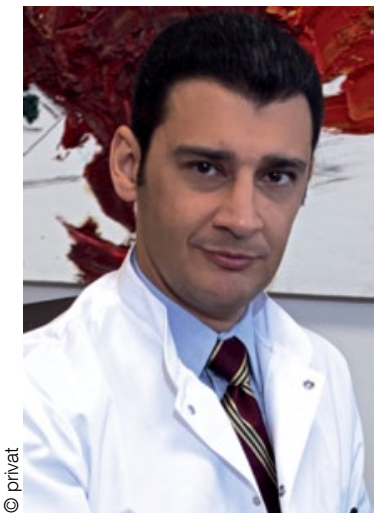

„Wir haben ein sehr gutes Gesundheitssystem. Aber was bei uns ein bisschen fehlt, ist die Zusammenarbeit"

\section{Doz. Dr. Afshin Assadian}

wissenschaftlicher Sprecher GFÖ

Die regelmäßige ärztliche Kontrolle nach Abschluss der Behandlung ist ebenfalls wichtig, weil ein hoher Anteil der Patienten sehr lange unter chronischen Wunden - etwa ein bis fünf Jahre - leidet und viele wiederholt daran erkranken. Risikogruppen sollten neben einem gesunden Lebensstil und der Reduktion von Risikofaktoren auch auf eine gute Fußpflege achten. „Häufig werden chronische Wunden an den Füßen von älteren Menschen aufgrund ihrer eingeschränkten Gelenkigkeit nicht rechtzeitig bemerkt. Oft trauen sie sich auch nicht den Schmerz anzusprechen", so Trautinger. GFÖ-Geschäftsführer Mag. Marcus Wilding, ergänzt: „Es ist ganz wichtig, dass auch Familienmitglieder dafür ein Auge haben. Es könnte durchaus sein, dass die Oma oder der Opa klagen, dass sie über einen längeren Zeitraum hinweg nur schlecht gehen können. Dann sollte man eben genauer nachfragen und nachschauen, woran das liegt." $\quad$ ÄWO

Quelle: Pressegespräch „Volksleiden,Chronische Wunde - Gefäßforum Österreich startet neue Gesundheitskampagne“, Wien, 16. Oktober 2012 